\title{
BMJ Open Examining the barriers and facilitators for Māori accessing injury and rehabilitation services: a scoping review protocol
}

\author{
Vicky Nelson (D) , ${ }^{1}$ Michelle Lambert, ${ }^{1}$ Lauralie Richard (D) , ${ }^{2}$ Sarah Derrett, ${ }^{1}$ \\ Emma Wyeth ${ }^{1}$
}

To cite: Nelson V, Lambert M, Richard L, et al. Examining the barriers and facilitators for Māori accessing injury and rehabilitation services: a scoping review protocol. BMJ Open 2022;12:e048252. doi:10.1136/ bmjopen-2020-048252

- Prepublication history and additional supplemental material for this paper are available online. To view these files, please visit the journal online (http://dx.doi.org/10.1136/ bmjopen-2020-048252).

Received 28 January 2021 Accepted 08 September 2021

Check for updates

(c) Author(s) (or their employer(s)) 2022. Re-use permitted under CC BY-NC. No commercial re-use. See rights and permissions. Published by BMJ.

${ }^{1} \mathrm{Ngāi}$ Tahu Māori Health Research Unit, Preventive and Social Medicine, University of Otago, Dunedin Campus, Dunedin, New Zealand ${ }^{2}$ General Practice and Rural Health, Dunedin School of Medicine, University of Otago, Dunedin, New Zealand

Correspondence to Associate Professor Emma Wyeth;

emma.wyeth@otago.ac.nz

\section{ABSTRACT}

Introduction Injury accounts for $10 \%$ of the global burden of disease. While the literature is scarce, research investigating injury among Indigenous populations has found incidence and prevalence rates are higher, compared with non-Indigenous populations. New Zealand is no exception; Māori have higher rates of injury and disability compared with non-Māori. Given the burden of injury for Māori, this scoping review aims to identify, understand and map available literature related to the barriers and facilitators to accessing injury-related healthcare for Māori in New Zealand.

Methods and analysis A scoping review will be conducted to identify the relevant literature and provide an opportunity to highlight key concepts and research gaps in the literature. This work will be guided by the scoping review framework developed by Arksey and 0'Malley and will be underpinned by Kaupapa Māori research principles. The overall project is also be guided by a Māori advisory group. Database searches, for example, MEDLINE (Ovid), Scopus and Embase, will be used to identify empirical literature, and Google, New Zealand government websites and relevant non-government organisations will be used to identify relevant grey literature.

Ethics and dissemination To the best of our knowledge, this scoping review is the first to systematically examine the currently available literature relating to the barriers and facilitators of accessing injury-related healthcare for Māori in New Zealand. Ethical approval was not required for this scoping review. Dissemination will include publication of the scoping review findings in a peer-reviewed journal, as well as presentations at conferences, to the project's advisory group, and staff working in the field of Māori disability and rehabilitation.

\section{INTRODUCTION}

Injury accounts for $10 \%$ of the global burden of disease. While the literature is scarce, research investigating injury among Indigenous populations shows, like many other health-related issues, that incidence and prevalence rates are higher for Indigenous populations compared with non-Indigenous populations. $^{2-4}$ Māori, the Indigenous

\section{Strengths and limitations of this study}

- To the best of the authors' knowledge, this is the first comprehensive scoping review to investigate barriers and facilitators of accessing injury treatment and rehabilitation for Māori and will help ensure a better understanding of the current context.

- Te reo Māori (Māori language) sources will be included in the grey literature review to ensure we capture a complete picture.

- A limitation of this study is that the findings for Māori in New Zealand may not be generalisable to other Indigenous populations, although we would expect there would be some relevance, especially given the limited literature available.

peoples of New Zealand, are no exception, experiencing a greater injury burden than non-Māori. ${ }^{5-7}$ Māori experience higher prevalence of injury and disability due to injury, ${ }^{8}$ higher hospitalisation rates for injury, ${ }^{910}$ and higher morbidity and mortality rates due to injury ${ }^{8}$ compared with non-Māori. The estimated years of life lost due to injury for Māori are twice those for non-Māori. ${ }^{11}$ Similar disparities are also seen for other Indigenous populations globally. ${ }^{3} 41213$ Despite these burdens, little research appears to have investigated a range of injury types, and predictors of post-injury outcomes, specifically for Māori and other Indigenous populations.

The Prospective Outcomes of Injury Study (POIS) investigated injury and postinjury outcomes in New Zealand. ${ }^{14} 15$ POIS is a longitudinal study that recruited 2856 people injured between 2007 and 2009 who received an entitlement claim from New Zealand's no-fault injury insurer, the Accident Compensation Corporation (ACC). Further, POIS was intentionally designed to enable specific analyses and meaningful and appropriate findings for injured 
Māori. ${ }^{15} 16$ POIS has shown that Māori experience poorer post-injury outcomes compared with non-Māori, with $50 \%$ of Māori experiencing disability three months postinjury. ${ }^{17}$ Further, at 12 months post-injury, Māori experience greater disability, mobility issues and psychological distress compared with non-Māori. ${ }^{18}$ Approximately $50 \%$ of Māori participants also reported experiencing pain and discomfort, and were more likely to experience a subsequent injury in the first 12 months after an initial injury. ${ }^{18}$ Alarmingly, adverse postinjury outcomes for Māori persist longer term, with Māori hospitalised for injury having a $64 \%$ increased risk of disability 24 months postinjury. ${ }^{9}{ }^{19}$

Given these injury burdens and poor post-injury outcomes, equitable access to, and engagement with, appropriate and timely healthcare services is imperative for improved injury outcomes and experiences. ${ }^{20} 21$ However, evidence highlights that Māori experience differential access and use of healthcare and rehabilitation services. $^{20}$ 22-24 Māori experience lower rates of general practitioner access, ${ }^{22}$ differential use of surgery services, ${ }^{24}$ longer hospital stays ${ }^{24}$ disparities in social and rehabilitation services access, ${ }^{24}{ }^{25}$ and lower rates of access to ACC and rehabilitation entitlements. ${ }^{25} 26$ Even with subsidised healthcare options, Māori have lower rates of access to healthcare and rehabilitation services, suggesting that cost is not the only barrier. ${ }^{22}$

Reduced access to healthcare services contributes to poor health outcomes. ${ }^{27}$ In previous research, Māori who reported trouble accessing healthcare services for injury were found to have increased risk of longer-term disability after injury. ${ }^{19}$ Additionally, ACC has reported differential service use by Māori and has identified that social, cultural, economic and geographical barriers contribute to this. ${ }^{26}$ The existing literature, including highlighting the inequities in injury prevalence, morbidity and mortality, ${ }^{811}$ adverse outcomes after injury ${ }^{17-1928} 29$ and access to injury-related care experienced by Māori, ${ }^{19} 252629$ indicate a need to further understand the difficulties that injured Māori face when accessing healthcare services.

The aim of this scoping review is to identify current literature about the barriers and facilitators of accessing injury and rehabilitation healthcare services by injured Māori in New Zealand and Indigenous populations globally. Specific objectives are to identify the extent and nature of the available literature, to summarise the main findings related to the barriers and facilitators of accessing injury and rehabilitation healthcare services by Māori and other Indigenous populations, and to identify any gaps in the literature.

\section{METHODS AND ANALYSIS \\ Concepts and definitions}

Defining and understanding access to healthcare can be challenging and complex. ${ }^{21}$ There are varying interpretations of the term 'access' across the literature. ${ }^{21} 30$ Often the term access has been used to describe characteristics of services at the initial point of contact or the ability for healthcare-seeking individuals to make that initial point of contact. ${ }^{30}$ This view is largely individualised and fails to recognise the complexity of accessing appropriate healthcare. ${ }^{2022}{ }^{30}$ Further, such views largely ignore the holistic views of Indigenous peoples. ${ }^{20} 22$

A conceptualised framework defining access, which incorporates a patient-centred perspective of access, has been developed by Levesque $e t a .^{30}$ The framework defines access as "the opportunity to reach and obtain appropriate healthcare services, in situations of perceived need of care"..$^{30}$ This considers the characteristics of the health systems, the characteristics of the populations that seek healthcare, and the processes and pathways that link the two sides so that access is achieved..$^{21} 30$

For this scoping review, we will use Levesque et $a l \mathrm{~s}^{30}$ framework to define and conceptualise access to injury and rehabilitation services. The framework (see figure 1) depicts dimensions of access and healthcare use, along two parallel sides: the supply side of the healthcare system, and the population who seek access to healthcare services. There are five dimensions that correspond to the supply of care and access by the healthcare system: (1) approachability, (2) acceptability, (3) availability and accommodation, (4) affordability, and (5) appropriateness. ${ }^{30}$ The framework also includes five dimensions corresponding to the characteristics of the population seeking access to healthcare. These dimensions include (1) ability to perceive, (2) ability to seek, (3) ability to reach, (4) ability to pay, and (5) ability to engage. ${ }^{30}$ The combined dimensions are not independent and therefore influence each other. ${ }^{30}$ This framework lends itself to investigating the cause of inequities at the individual level, but also at system and structural levels.

To understand the available literature regarding the barriers and facilitators to accessing injury-related healthcare for Māori in New Zealand and other Indigenous populations globally, we will conduct a scoping review. A scoping review was deemed the most appropriate method to identify available literature and "map the literature on a particular topic or research area and provide an opportunity to identify key concepts; gaps in the research". 3132 Scoping reviews, unlike systematic reviews, do not require the appraisal or synthesis of the review findings. ${ }^{32} 33$ Therefore, a scoping review allows for the inclusion of a broad range of study designs, from both the published and grey literature, while still employing a standardised and rigorous process. ${ }^{32} 33$

The protocol outlined for this scoping review will be guided by the scoping review framework developed by Arksey and O'Malley, ${ }^{32}$ and the framework update outlined by Levac et al. ${ }^{31}$ Specifically, the framework sets out six stages to follow during a scoping review: (1) identifying the research question, (2) identifying the relevant literature, (3) study selection, (4) charting the data, (5) collating, summarising and reposting the findings, and (6) consultation. ${ }^{31} 32$

Further, this project is underpinned by Kaupapa Māori principles, ${ }^{34} 35$ putting Māori views and realities at the 


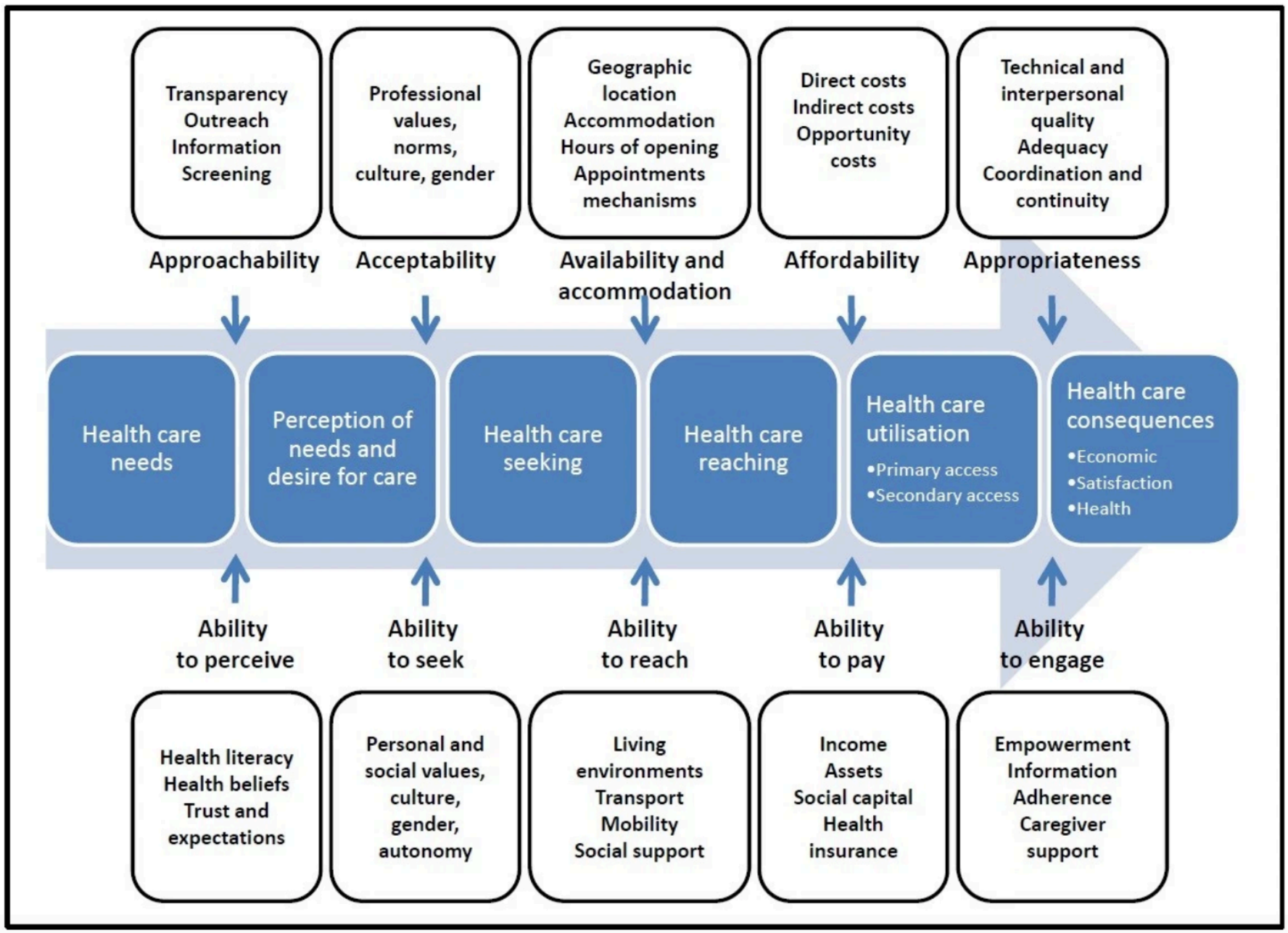

Figure 1 Conceptual framework of access to healthcare (reproduced from Levesque et al ${ }^{30}$ ).

centre of the research; it is research 'by Māori, with Māori, for Māori'. ${ }^{34} 35$

\section{Stage 1: defining the research questions}

The research questions guiding our scoping review were developed through a collaborative approach with the research team and the Māori advisory group. Two research questions have been developed to guide the search of the published and grey literatures separately. We are interested in scoping the available published literature pertaining to the barriers and facilitators of accessing injury and rehabilitation care for Māori and other Indigenous populations. We have limited the grey literature search to Māori only as we are specifically interested in the policies, structures and systems that influence healthcare access for injured Māori in New Zealand.

Research question to be answered by the published literature search

What are the barriers and facilitators to accessing injury and rehabilitation services for Māori and other Indigenous populations?
Research question to be answered by the grey literature search What are the barriers and facilitators to accessing injury and rehabilitation services for Māori in New Zealand?

\section{Stage 2: identifying relevant studies}

Separate search strategies will be developed to comprehensively search both the empirical literature and the grey literature to address our research questions. Identifying relevant literature in this scoping review will include four steps.

An initial search by a single reviewer, in collaboration with a subject librarian from the University of Otago, has been conducted in MEDLINE (Ovid). Keywords, text words, Medical Subject Headings (MeSH) terms, index terms, titles and abstracts were analysed to identify relevant search terms and keywords for the development of an appropriate search strategy for all databases included in this scoping review. The identified terms were then reviewed by the wider research team, and the search strategy was finalised. Second, a search using all identified keywords appropriate to each database relevant to this review was conducted (see table 1 for MEDLINE search strategy; see online supplemental file 1 for Scopus and 


\begin{tabular}{|c|c|c|}
\hline & Terms & Results \\
\hline 1 & $\begin{array}{l}\text { Maori OR "New Zealand Indigenous" OR } \\
\text { Indigenous OR First Nations OR Aborigin* } \\
\text { OR Native.af. }\end{array}$ & 259526 \\
\hline 2 & $\begin{array}{l}\text { Injury OR injur OR trauma OR Wound OR } \\
\text { Accident }\end{array}$ & 1632314 \\
\hline 3 & $\begin{array}{l}\text { "health services" OR "injur* services" } \\
\text { OR "injur* providers" OR rehabilitat" } \\
\text { OR physiotherap* OR health care OR } \\
\text { healthcare.af. }\end{array}$ & 1945052 \\
\hline 4 & $\begin{array}{l}\text { experiences or access or issues or } \\
\text { engagement or affordabil }{ }^{\star} \text { or acceptabl } \\
\text { or appropriat }{ }^{\star} \text { or availabilit* or enable }{ }^{\star} \text { or } \\
\text { factor }^{\star} \text {.af. }\end{array}$ & 7291075 \\
\hline 5 & 1 AND 2 AND 3 AND 4 & 243 \\
\hline 6 & $\begin{array}{l}\text { Limit } 5 \text { to (full text AND "all adult (18 years } \\
\text { plus)" AND Humans AND English }\end{array}$ & 22 \\
\hline
\end{tabular}

Embase search strategies). Third, the reference lists and 'cited by' function will be used to search for any other relevant studies for inclusion. Lastly, our advisory group will be asked to identify additional relevant literature for inclusion in this review.

The databases to be searched to identify empirical literature will include MEDLINE (Ovid), Scopus, Embase, CINAHL Complete and Cochrane library. In addition, Google, New Zealand government websites and relevant non-government organisations and other organisations' websites identified through our own networks and collaboration with our advisors will be searched to identify relevant grey literature. Searches will be limited to studies published in the English language, open-access journals and those that include adults (18 years and above). For the grey literature, searches will be further limited to literature published from the year 2000 to present.

\section{Stage 3: study selection}

The search strategy outlined previously will be conducted by a single researcher. All identified literature will be exported into Endnote V.X8.2; duplicated studies will be removed; and the Endnote library will be shared with a second reviewer. Titles, abstracts and full texts will be independently screened for eligibility for the data extraction (stage 4). An initial pilot of the screening process for 5-10 studies, using the subsequent criteria, will be conducted. Then, the remaining screening and study selection process will be completed by two researchers independently and compared. Where there are inconsistencies, a third researcher will be consulted. A University of Otago subject librarian will be consulted if difficulties arise. Once completed, the final number of studies included for data extraction will be revealed. The study selection process will be summarised and reported using a Preferred Reporting Items for Systematic Reviews and Meta-Analyses (PRISMA) chart and PRIMSA extension for scoping reviews (PRISMA-ScR). ${ }^{36}$ The inclusion and exclusion criteria for both the published and grey literature are further discussed.

\section{Criteria for the published literature}

Titles and abstracts of empirical studies will be included if they refer to (1) an Indigenous population, (2) injury or rehabilitation or healthcare or health services, and (3) barriers or facilitators of healthcare, or healthcare access, or healthcare experiences.

Titles and abstracts will be excluded if they: (1) do not include an Indigenous population, (2) do not include injury, or if they refer to other non-injury medical conditions, illnesses or diseases, (3) do not refer to healthcare access or experiences, and (4) are books, book chapters or theses.

\section{Criteria for the grey literature}

Titles and abstracts of grey literature will be included for data extraction if they refer to: (1) Māori, (2) injury or rehabilitation or injury and rehabilitation healthcare or services, and (3) healthcare access, barrier or facilitators or experiences.

Titles and abstracts will be excluded from data extraction if they: (1) do not refer to or include Māori, (2) do not include injury or rehabilitation, or injury and rehabilitation healthcare or services, (3) do not include healthcare access or experiences, (4) are bulletins, newsletters, conference abstracts or proceedings, lectures, or non-written material, and (5) published before the year 2000.

\section{Stage 4: data extraction and charting}

A data extraction sheet and charting guidelines will be developed collaboratively by the research team, guided by the access framework and the scoping review research questions. Once finalised, the data extraction sheet will be piloted, as described previously.

Data extraction and charting will be a two-step process. First, descriptive details of each study will be recorded in a pre-defined extraction chart in Microsoft Excel v.16. Second, all sources will be imported into qualitative data management software, NVivo. ${ }^{37}$ Studies will be analysed and coded using the pre-defined coding guideline. ${ }^{30}$ Any additional codes will be discussed and agreed by the two researchers.

Further, data extraction, including the pre-defined extraction chart and NVivo coding, will adhere to the criteria that are set out in the Consolidated Criteria for Strengthening Reporting of Health Research Involving Indigenous Peoples: The (CONSIDER) Statement, developed by Huria $e t a l .^{38}$ The Statement includes a checklist of eight domains, comprising seven criteria for the 'strengthening the reporting of health research involving Indigenous peoples', and aiding the 'advancement of Indigenous health outcomes and development'. ${ }^{38}$ The domains include: (1) research governance, (2) prioritisation, (3) relationships with Indigenous stakeholders and 
research team, (4) methodologies, (5) participation, (6) capacity, (7) analysis and interpretation, and (8) dissemination. Additionally, we will include further details of how this Statement has informed the different stages of the scoping review in the manuscript that presents the scoping review findings.

Once data extraction and charting are completed, findings will be compared, and the wider research team will be consulted where there are discrepancies.

\section{Stage 5: collating, summarising and reporting findings}

The aim of this scoping review is to map the existing literature and to summarise the findings presented across the studies included in the review. The extracted data will be presented according to the different dimensions of the access framework. ${ }^{30}$ A descriptive summary of all included studies, as well as a narrative summary of the findings and how these relate to the reviews' aims and research questions, will also accompany the data presented.

Further, the scoping review, particularly the collating and reporting of results, will be informed by Kaupapa Māori research principles, as well as the CONSIDER statement. ${ }^{38}$ Kaupapa Māori is a methodology which privileges Māori "voices and perspectives, methodologies and analysis, whereby Māori realities are seen as legitimate" ${ }^{\prime 39}$ (p.50). As with the CONSIDER statement, we will include further details of how these research principles informed the different stages of the scoping review in the manuscript that presents the actual scoping review findings.

\section{Stage 6: consultation}

Scoping reviews can be enhanced if practitioners and consumers contribute to the work. ${ }^{31}{ }^{32}$ While encouraged, but optional, in the Arksey and O'Malley methodology, ${ }^{32}$ it is a fundamental component of kaupapa Māori research, ${ }^{34}{ }^{35}$ and the CONSIDER statement and checklist, ${ }^{38}$ and will be included in this scoping review. Specifically, this scoping review is supported by an advisory group, with members from a range of organisations with a wealth of expertise relating to Māori health, health services, injury and rehabilitation. To date, the advisory group has been consulted to discuss the scoping review aims, strategy and search terms. The advisory group will continued to be consulted throughout the scoping review stages.

\section{ETHICS AND DISSEMINATION}

Ethical approval was not required for this scoping review. To the best of our knowledge, this scoping review is the first to systematically map the currently available literature concerning the barriers and facilitators to accessing injury related healthcare for Māori in New Zealand and Indigenous populations globally. Therefore, this scoping review will be of interest to international researchers (e.g., health, Indigenous), injury and rehabilitation-related clinicians, key New Zealand organisations (eg, ACC, the Ministry of Health) and Māori health organisations. Our dissemination strategy will include the publication of the scoping review findings in a peer-reviewed journal, presentations to people working in the field of the Māori disability and rehabilitation. This scoping review is the first phase of a larger project that includes a Māori disability and rehabilitation service mapping exercise in the Ngai Tahu takiwā and interviews with both health professionals with expertise in the field of injury rehabilitation, as well as with and injured Māori.

\section{PATIENT AND PUBLIC INVOLVEMENT}

No patients were involved in the design of the study, but the majority of representatives on the study advisory group are regularly involved in patient-facing activities.

Twitter Emma Wyeth @ehwyeth

Acknowledgements We acknowledge Christy Ballard, the University of Otago, Department of Preventive and Social Medicine subject librarian, for her assistance in the development of the search strategy and related terms.

Contributors VN completed an initial draft of the scoping review protocol and led the development of the research questions and methods. ML was responsible for the review of the scoping review development and processes, as well as provided feedback on multiple iterations of this article. EW and SD jointly helped conceptualise the scoping review and guided the development of the research questions and methods, and provided feedback on multiple iterations of this article. LR was consulted on the scoping review strategy and provided feedback on multiple iterations of this article.

Funding This work was supported by a 2019 University of Otago Research Grant. Competing interests None declared.

Patient consent for publication Not applicable.

Provenance and peer review Not commissioned; externally peer reviewed.

Supplemental material This content has been supplied by the author(s). It has not been vetted by BMJ Publishing Group Limited (BMJ) and may not have been peer-reviewed. Any opinions or recommendations discussed are solely those of the author(s) and are not endorsed by BMJ. BMJ disclaims all liability and responsibility arising from any reliance placed on the content. Where the content includes any translated material, BMJ does not warrant the accuracy and reliability of the translations (including but not limited to local regulations, clinical guidelines, terminology, drug names and drug dosages), and is not responsible for any error and/or omissions arising from translation and adaptation or otherwise.

Open access This is an open access article distributed in accordance with the Creative Commons Attribution Non Commercial (CC BY-NC 4.0) license, which permits others to distribute, remix, adapt, build upon this work non-commercially, and license their derivative works on different terms, provided the original work is properly cited, appropriate credit is given, any changes made indicated, and the use is non-commercial. See: http://creativecommons.org/licenses/by-nc/4.0/.

ORCID iDs

Vicky Nelson http://orcid.org/0000-0002-2765-9601

Lauralie Richard http://orcid.org/0000-0002-2497-3857

\section{REFERENCES}

1 Haagsma JA, Graetz N, Bolliger I, et al. The global burden of injury: incidence, mortality, disability-adjusted life years and time trends from the global burden of disease study 2013. Inj Prev 2016;22:3-18

2 Abdalla S, Kelleher CC, Quirke B, et al. Disparities in fatal and nonfatal injuries between Irish travellers and the Irish general population are similar to those of other indigenous minorities: a cross-sectional population-based comparative study. BMJ Open 2013;3:e002296.

3 Karmali S, Laupland K, Harrop AR, et al. Epidemiology of severe trauma among status Aboriginal Canadians: a population-based study. CMAJ 2005;172:1007-11. 
4 Soininen L, Pukkala E. Mortality of the SAMI in northern Finland 1979-2005. Int J Circumpolar Health 2008;67:45-57.

5 Reid P, Robson B. Understanding health inequities. In: Robson B, Harris R, eds. Hauora: Mãori standards of health IV. A study of the years 2000-2005. Wellington, NZ: te Rōpū Rangahau Hauora a Eru Pōmare, 2007: 3-10.

6 Ratima K, Ratima M. Māori Experience of Disability and Dlsability Support Services. In: Robson B, Harris R, eds. Hauora: Māori standards of health IV. A study of the years 2000-2005. Te Rōpū Rangahau Hauora a Eru Pōmare: Wellington, New Zealand, 2007: 189-208.

7 Ministry of Health, Tatau Kahukura. Māori health chart book 2010. 2nd edn. Wellington: Ministry of Health, 2010.

8 Statistics New Zealand. He Hauā Māori - findings from the 2013 disability survey. Statistics New Zealand, 2015.

9 Derrett S, Wilson S, Samaranayaka A, et al. Prevalence and predictors of disability 24-months after injury for hospitalised and non-hospitalised participants: results from a longitudinal cohort study in New Zealand. PLoS One 2013;8:e80194.

10 Office for Disability Issues and Statistics New Zealand. Disability and Māori in New Zealand in 2006: results from the New Zealand disability survey. Wellington, NZ: Statistics New Zealand, 2010.

11 Ministry of Health and Accident Compensation Corporation. Injury-related health loss: a report from the New Zealand burden of diseases, injuries and risk factors study 2006-2016. Wellington: Ministry of Health, 2013.

12 Hassler S, Johansson R, Sjölander P, et al. Causes of death in the SAMI population of Sweden, 1961-2000. Int J Epidemiol 2005;34:623-9.

13 Vos T, Barker B, Begg S, et al. Burden of disease and injury in Aboriginal and Torres Strait Islander peoples: the Indigenous health gap. Int J Epidemiol 2009;38:470-7.

14 Derrett S, Langley J, Hokowhitu B, et al. Prospective outcomes of injury study. Inj Prev 2009;15:e3.

15 Derrett S, Davie G, Ameratunga S, et al. Prospective outcomes of injury study: recruitment, and participant characteristics, health and disability status. Inj Prev 2011;17:415-8.

16 Wyeth EH, Derrett S, Hokowhitu B, et al. Rangatiratanga and Oritetanga: responses to the Treaty of Waitangi in a New Zealand study. Ethn Health 2010;15:303-16.

17 Maclennan B, Wyeth E, Hokowhitu B, et al. Injury severity and 3-month outcomes among Māori: results from a New Zealand prospective cohort study. N Z Med J 2013;126:39-49.

18 Maclennan B, Wyeth E, Davie G, et al. Twelve-month post-injury outcomes for Māori and non-Māori: findings from a New Zealand cohort study. Aust N Z J Public Health 2014;38:227-33.

19 Wyeth EH, Samaranayaka A, Davie G, et al. Prevalence and predictors of disability for Māori 24 months after injury. Aust N Z J Public Health 2017;41:262-8.

20 Ellison-Loschmann L, Pearce N. Improving access to health care among New Zealand's Maori population. Am J Public Health 2006;96:612-7.

21 Hamm LM, Black J, Burn H, et al. Interventions to promote access to eye care for non-Indigenous, non-dominant ethnic groups in high-income countries: a scoping review protocol. BMJ Open 2020;10:e033775.

22 Jansen P. Non-financial barriers to primary health care services for Maori. J Prim Health Care 2009;1:240.

23 Reid J, Cormack D, Crowe M. The significance of socially-assigned ethnicity for self-identified Māori accessing and engaging with primary healthcare in New Zealand. Health 2016;20:143-60.

24 Jansen P, Bacal K, Crengle S. He Ritenga Whakaaro: Māori experiences of health services. Auckland: Mauri Ora Associates, 2008.

25 Jansen D. Māori experience of ACC - final report for department of labour. Auckland: Mauri Ora Associates, 2010.

26 Accident Compensation Corporation. 1.0 Whāia te Tika: Māori responsiveness reports. Wellington, New Zealand: Accident Compensation Corporation, 2017.

27 Reid J, Cormack D, Crowe M. The significance of relational continuity of care for Māori patient engagement with predominantly non-Māori doctors: findings from a qualitative study. Aust $N Z$ J Public Health 2016;40:120-5

28 Wyeth EH, Derrett S, Hokowhitu B, et al. Indigenous injury outcomes: life satisfaction among injured Māori in New Zealand three months after injury. Health Qual Life Outcomes 2013;11:120.

29 Wyeth $\mathrm{EH}$, Samaranayaka A, Lambert M, et al. Understanding longer-term disability outcomes for Māori and non-Māori after hospitalisation for injury: results from a longitudinal cohort study. Public Health 2019;176:118-27.

30 Levesque J-F, Harris MF, Russell G. Patient-centred access to health care: conceptualising access at the interface of health systems and populations. Int J Equity Health 2013;12:18.

31 Levac D, Colquhoun H, O'Brien KK. Scoping studies: advancing the methodology. Implement Sci 2010;5:69.

32 Arksey H, O'Malley L. Scoping studies: towards a methodological framework. Int J Soc Res Methodol 2005;8:19-32.

33 Peters MDJ, Godfrey CM, Khalil H, et al. Guidance for conducting systematic scoping reviews. Int $J$ Evid Based Healthc 2015;13:141-6.

34 Cram F. Rangahau Māori: Tona tika, tona pono - The Validity and Integrity of Māori Research, in Research Ethics in Aotearoa New Zealand. Auckland, New Zealand: Pearson Education New Zealand Limited, 2001: 35-52.

35 Jones Bet al. Whānau Tuatahi: Māori community partnership research using a Kaupapa Māori methodology. Mai Rev 2010;3:1-14.

36 Tricco AC, Lillie E, Zarin W, et al. PRISMA extension for scoping reviews (PRISMA-ScR): checklist and explanation. Ann Intern Med 2018;169:467-73.

37 Bergin M. NVivo 8 and consistency in data analysis: reflecting on the use of a qualitative data analysis program. Nurse Res 2011;18:6-12.

38 Huria T, Palmer SC, Pitama S, et al. Consolidated criteria for strengthening reporting of health research involving Indigenous peoples: the consider statement. BMC Med Res Methodol 2019;19:173.

39 Cram F. Talking ourselves up. AlterNative: Int J Indigen People 2006;2:28-43. 